\title{
Diagnostic Performance of Fluorine-18-Fluorodeoxyglucose Positron Emission Tomography in the Postchemotherapy Management of Patients with Seminoma: Systematic Review and Meta-Analysis
}

\author{
Giorgio Treglia, ${ }^{1}$ Ramin Sadeghi, ${ }^{2}$ Salvatore Annunziata, ${ }^{3}$ Carmelo Caldarella, ${ }^{3}$ \\ Francesco Bertagna, ${ }^{4}$ and Luca Giovanella ${ }^{1}$ \\ ${ }^{1}$ Department of Nuclear Medicine and PET/CT Center, Oncology Institute of Southern Switzerland, Via Ospedale 12, \\ 6500 Bellinzona, Switzerland \\ ${ }^{2}$ Nuclear Medicine Research Center, Mashhad University of Medical Sciences, Mashhad, Iran \\ ${ }^{3}$ Institute of Nuclear Medicine, Catholic University of the Sacred Heart, 00168 Rome, Italy \\ ${ }^{4}$ Department of Nuclear Medicine, University of Brescia and Spedali Civili, 25123 Brescia, Italy
}

Correspondence should be addressed to Giorgio Treglia; giorgiomednuc@libero.it

Received 8 February 2014; Accepted 11 April 2014; Published 15 May 2014

Academic Editor: Yukio Kageyama

Copyright (C) 2014 Giorgio Treglia et al. This is an open access article distributed under the Creative Commons Attribution License, which permits unrestricted use, distribution, and reproduction in any medium, provided the original work is properly cited.

\begin{abstract}
Objective. To meta-analyze published data about the diagnostic performance of fluorine-18-Fluorodeoxyglucose $\left({ }^{18} \mathrm{~F}-\mathrm{FDG}\right)$ positron emission tomography (PET) and PET/computed tomography (PET/CT) in the postchemotherapy management of patients with seminoma. Methods. A comprehensive literature search of studies published through January 2014 on this topic was performed. All retrieved studies were reviewed and qualitatively analyzed. Pooled sensitivity and specificity, positive and negative predictive values (PPV and NPV), accuracy, and area under the summary ROC curve (AUC) of ${ }^{18}$ F-FDG-PET or PET/CT on a per examination-based analysis were calculated. Subgroup analyses considering the size of residual/recurrent lesions were carried out. Results. Nine studies including 375 scans were selected. The pooled analysis provided the following results: sensitivity $78 \%$ (95\% confidence interval (95\% CI): 67-87\%), specificity 86\% (95\% CI: 81-89\%), PPV 58\% (95\% CI: 48-68\%), NPV 94\% (95\% CI: 90$96 \%$ ), and accuracy $84 \%$ (95\% CI: $80-88 \%$ ). The AUC was 0.90 . A better diagnostic accuracy of ${ }^{18}$ F-FDG-PET or PET/CT in evaluating residual/recurrent lesions $>3 \mathrm{~cm}$ compared to those $<3 \mathrm{~cm}$ was found. Conclusions. ${ }^{18} \mathrm{~F}-\mathrm{FDG}$-PET and PET/CT were demonstrated to be accurate imaging methods in the postchemotherapy management of patients with seminoma; nevertheless possible sources of false-negative and false-positive results should be considered. The literature focusing on this setting still remains limited and cost-effectiveness analyses are warranted.
\end{abstract}

\section{Introduction}

Seminoma is a malignant germ cell tumor of the testis or, more rarely, of extragonadal locations which originates in the germinal epithelium of the seminiferous tubules. About half of germ cell tumors of the testis are seminomas. Metastatic seminoma is the paradigm of a curable cancer and the cure rate has now attained 95\%, with extrapulmonary visceral metastases being the main identified prognostic factor $[1,2]$. The optimal postchemotherapy management of patients with seminoma has been widely debated. In fact, postchemotherapy residual lesions at morphological imaging are frequent and surgical resection of these findings usually reveals necrosis or fibrosis [3]. Some centers suggested performing surgery for all residual lesions $>3 \mathrm{~cm}$ because the likelihood of viable tumor increases in residual masses larger than $3 \mathrm{~cm}$, while others suggested observing and using salvage treatment only if the lesions fail to shrink or for clearly documented relapse taking into account the technical difficulties and potential morbidity of surgery [3]. 
Over the past 2 decades, the postchemotherapy management of patients with seminoma has evolved due to the increasing use of fluorine-18 fluorodeoxyglucose positron emission tomography $\left({ }^{18} \mathrm{~F}\right.$-FDG-PET). This method alone or combined with computed tomography (PET/CT) has been proposed as noninvasive tool to assess the disease extent in cancer patients. Since ${ }^{18}$ F-FDG is a glucose analogue, this radiopharmaceutical may be very useful in detecting malignant lesions which usually present high glucose metabolism [4]. Hybrid PET/CT device allows enhanced detection and characterization of neoplastic lesions, by combining the functional data obtained by PET with morphological data obtained by CT [4]. The incorporation of noninvasive imaging modalities, such as ${ }^{18}$ F-FDG-PET or PET/CT, into the management algorithm may allow better delineation of the presence of viable residual tumor and thus allow better risk stratification in patients with seminoma $[2,3]$.

Several prospective and retrospective studies evaluated the diagnostic performance of ${ }^{18}$ F-FDG-PET or PET/CT in the postchemotherapy management of patients with seminoma reporting conflicting results. The aim of our study is to perform an updated systematic review and meta-analysis in order to provide more evidence-based data in this setting.

\section{Methods}

This systematic review and meta-analysis were performed according to the "Preferred Reporting Items for Systematic Reviews and Meta-Analyses" (PRISMA) statement which describes an evidence-based minimum set of items for reporting in systematic reviews and meta-analyses [5].

2.1. Search Strategy. A comprehensive computer literature search of the PubMed/MEDLINE and Scopus databases was conducted to find relevant published articles on the diagnostic performance of ${ }^{18}$ F-FDG-PET or PET/CT in the postchemotherapy management of patients with seminoma. We used a search algorithm that was based on a combination of the following terms: (a) "PET" or "positron emission tomography" and (b) "seminoma" or "seminomatous" or "testis" or "testicular" or "germinal" or "germ cell". No beginning date limit was used; the search was updated until January 31, 2014. To expand our search, references of the retrieved articles were also screened for additional studies.

2.2. Study Selection. Studies or subsets in studies investigating the diagnostic performance of ${ }^{18} \mathrm{~F}$-FDG-PET or $\mathrm{PET} / \mathrm{CT}$ in the postchemotherapy management of patients with seminoma (including evaluation of residual masses after chemotherapy and restaging) were eligible for inclusion. The exclusion criteria were (a) articles not within the field of interest of this review; (b) review articles, editorials or letters, comments, and conference proceedings; (c) case reports or small case series (less than 10 patients with seminoma); (d) articles evaluating patients with seminoma at initial staging; (e) insufficient information to reassess sensitivity and specificity in the postchemotherapy management; (f) articles not in English language; (g) possible data overlap (in such cases the most complete article was included).

Three researchers independently reviewed the titles and abstracts of the retrieved articles, applying the inclusion and exclusion criteria mentioned above. Articles were rejected if they were clearly ineligible. The same three researchers then independently reviewed the full-text version of the remaining articles to determine their eligibility for inclusion. Disagreements were resolved in a consensus meeting.

2.3. Data Extraction. For each included study, information was collected concerning basic study (authors, journals and year of publication, country of origin, and study design), patient characteristics, and technical aspects (device used, radiopharmaceutical injected dose, time between ${ }^{18}$ F-FDG injection and image acquisition, image analysis, and applied reference standard). For each study the number of truepositive, false-positive, true-negative, and false-negative findings for ${ }^{18}$ F-FDG-PET or PET/CT was recorded on a per examination-based analysis considering the qualitative PET analysis (visual analysis) performed by the authors.

2.4. Quality Assessment. The 2011 Oxford Center for Evidence-Based Medicine checklist for diagnostic studies was used for quality assessment of the included studies [15]. This checklist has 5 major parts as follows: representative spectrum of the patients, consecutive patient recruitment, ascertainment of the gold standard regardless of the index test results, independent blind comparison between the gold standard and index test results, and enough explanation of the test to permit replication.

2.5. Statistical Analysis. Sensitivity, specificity, accuracy, positive and negative predictive value, positive and negative likelihood ratio (LR), and diagnostic odd ratio (DOR) of ${ }^{18} \mathrm{~F}$ FDG-PET or PET/CT in the postchemotherapy management of patients with seminoma were obtained from individual studies on a per examination-based analysis. A random effects model was used for statistical pooling of the data. Pooled data were presented with 95\% confidence intervals (95\% CI). An $I^{2}$ index was used to test for heterogeneity among the studies. The area under the summary ROC curve (AUC) was calculated to measure the accuracy of ${ }^{18} \mathrm{~F}-\mathrm{FDG}$ PET or PET/CT. For publication bias evaluation, funnel plots, Egger's regression intercept [16], and Duval and Tweedie's method [17] were used. Subgroup analyses on the diagnostic performance of ${ }^{18} \mathrm{~F}$-FDG-PET or PET/CT in patients with postchemotherapy residual/recurrent lesions at CT with size $<$ or $>3 \mathrm{~cm}$ were also carried out.

Statistical analyses were performed using Meta-DiSc statistical software version 1.4 (Unit of Clinical Biostatistics, Ramón y Cajal Hospital, Madrid, Spain) and Comprehensive Meta-Analysis (CMA) software version 2 (BioStat, Englewood, NJ, USA). 


\section{Results}

3.1. Literature Search. The comprehensive computer literature search from PubMed/MEDLINE and Scopus databases revealed 490 articles. Reviewing titles and abstracts, 481 articles were excluded: 371 were excluded because they were not in the field of interest of this review, 70 were excluded as reviews and editorials or letters, 29 were excluded as case reports or small case series, 4 were excluded as articles evaluating patients with seminoma at initial staging, 3 were excluded for insufficient information to reassess sensitivity and specificity in the postchemotherapy management [1820], 2 articles were excluded because they were not in English language [21, 22], and 2 articles were excluded for possible data overlap $[23,24]$. Finally, nine articles including 375 patients were selected and were eligible for the systematic review and meta-analysis [6-14]; no additional studies were found screening the references of these articles (Figure 1). The characteristics of the included studies are presented in Tables 1, 2, 3, and 4 .

3.2. Qualitative Analysis (Systematic Review). Using the database search, 9 original articles written over the past 15 years were selected [6-14]. About the study design, three of these studies were prospective $[9,11,14], 5$ were retrospective $[6-8,10,13]$, and in one article this information was not provided [12]. Three studies were multicentric $[7,9,11]$, whereas six were monocentric $[6,8,10,12-14]$. Most of the patients performed ${ }^{18}$ F-FDG-PET or PET/CT for evaluating abdominal residual lesions at CT imaging after chemotherapy (Table 1).

Two studies used hybrid PET/CT $[6,8]$, whereas seven studies used PET only [7, 9-14]. Heterogeneous technical aspects between the included studies were found (Table 2). PET image analysis was performed by using qualitative criteria (visual analysis) in all the included studies [6-14] and adjunctive semiquantitative criteria (based on the calculation of the standardized uptake value (SUV)) in 3 out of 9 articles $[9,12,14]$.

The reference standard used to validate the ${ }^{18} \mathrm{~F}$-FDG-PET or PET/CT findings was quite different in the included studies (Table 4). The results of the quality assessment of the studies included in this meta-analysis, according to the 2011 Oxford Center for Evidence-Based Medicine checklist for diagnostic studies, are shown in Table 4.

Most of the studies included in this pooled analysis support the usefulness of ${ }^{18} \mathrm{~F}$-FDG-PET or PET/CT in the postchemotherapy management of patients with seminoma compared to CT alone [6-13]. Abdominal residual lesions at $\mathrm{CT}$ are quite frequent in patients with seminoma after chemotherapy and conventional imaging methods often do not discriminate between residual neoplastic lesions or fibrotic tissue, whereas ${ }^{18}$ F-FDG-PET may provide complementary metabolic information on these lesions. Furthermore, ${ }^{18}$ F-FDG-PET may detect early recurrent disease in patients with seminoma and normal CT findings $[6,13]$, because functional abnormalities may precede morphological changes. On the other hand, possiblesources of false- negative (small malignant lesions or with low proliferative index) and false-positive results (mainly inflammatory lesions) of ${ }^{18} \mathrm{~F}$-FDG-PET or PET/CT in the postchemotherapy management of patients with seminoma should be kept in mind [6-14].

About the impact on the clinical management a recent study demonstrated that ${ }^{18}$ F-FDG-PET or PET/CT may provide valuable information to this regard, particularly for clinical surveillance and posttherapy assessment and when relapse is suspected [6].

3.3. Quantitative Analysis (Meta-Analysis). The diagnostic performance results of ${ }^{18} \mathrm{~F}$-FDG-PET or PET/CT in the nine studies selected for the meta-analysis are presented in Figures 2-4 and reported below.

The sensitivity of ${ }^{18} \mathrm{~F}$-FDG-PET or PET/CT in the postchemotherapy management of patients with seminoma calculated on a per examination-based analysis ranged from $0 \%$ to $100 \%$, with pooled estimate of $78 \%$ (95\% CI: $67-$ $87 \%)$. The specificity of ${ }^{18} \mathrm{~F}-\mathrm{FDG}$-PET or PET/CT in the postchemotherapy management of patients with seminoma calculated on a per examination-based analysis ranged from $47 \%$ to $100 \%$, with pooled estimate of $86 \%$ (95\% CI: $81-$ $89 \%)$. The included studies were statistically heterogeneous in their estimate of sensitivity $\left(I^{2}: 66 \%\right)$ and specificity $\left(I^{2}\right.$ : 78\%) (Figure 2).

The pooled positive and negative predictive values and accuracy of these methods were 58\% (95\% CI: 48-68\%), 94\% (95\% CI: 90-96\%), and 84\% (95\% CI: 80-88\%), respectively.

The pooled positive LR, negative LR, and DOR were 4.59 (95\% CI: $2.6-8.3$ ), 0.26 (95\% CI: $0.09-0.71$ ), and 22.7 (95\% CI: 8.8-58.7), respectively (Figure 3). The AUC was 0.90 (Figure 2).

Egger's regression intercepts for sensitivity and specificity pooling were 0.7 (95\% CI: -1.3 to $2.7 ; P=0.43$ ) and 1.5 (95\% CI: -1 to $4 ; P=0.19$ ), respectively. Applying Duval and Tweedie's method, the funnel plot of sensitivity and specificity reached symmetry and the adjusted sensitivity and specificity decreased for $3.2 \%$ and $8.8 \%$, respectively (Figure 4 ).

Due to the statistical heterogeneity found in the calculation of the pooled sensitivity and specificity of ${ }^{18} \mathrm{~F}$ FDG-PET or PET/CT in the postchemotherapy management of patients with seminoma, subgroup analyses considering residual/recurrent lesions at CT with size $>$ or $<3 \mathrm{~cm}$ were performed, taking into account data provided by seven out of nine articles included in our meta-analysis. The subgroup analyses demonstrated that the pooled sensitivity and specificity of ${ }^{18} \mathrm{~F}$-FDG-PET or PET/CT were $47 \%$ (95\% CI: $21-73 \%$ ) and $89 \%$ (95\% CI: $82-94 \%$ ), respectively, for patients with postchemotherapy residual/recurrent lesions at CT $<3 \mathrm{~cm}$ and $89 \%$ (95\% CI: $75-97 \%)$ and $81 \%$ (95\% CI: 73-88\%), respectively, for patients with postchemotherapy residual/recurrent lesions at $\mathrm{CT}>3 \mathrm{~cm}$. Furthermore, the statistical heterogeneity largely decreased $\left(I^{2}\right.$ was $0 \%$ for sensitivity and specificity in both subgroup analyses). The AUC for patients with postchemotherapy residual/recurrent lesions at $\mathrm{CT}<$ or $>3 \mathrm{~cm}$ was 0.76 and 0.87 , respectively. 
490 records identified through database searching using the following terms:

(PET or positron emission tomography) and (seminoma or seminomatous or testis or testicular or germinal, or germ cell)

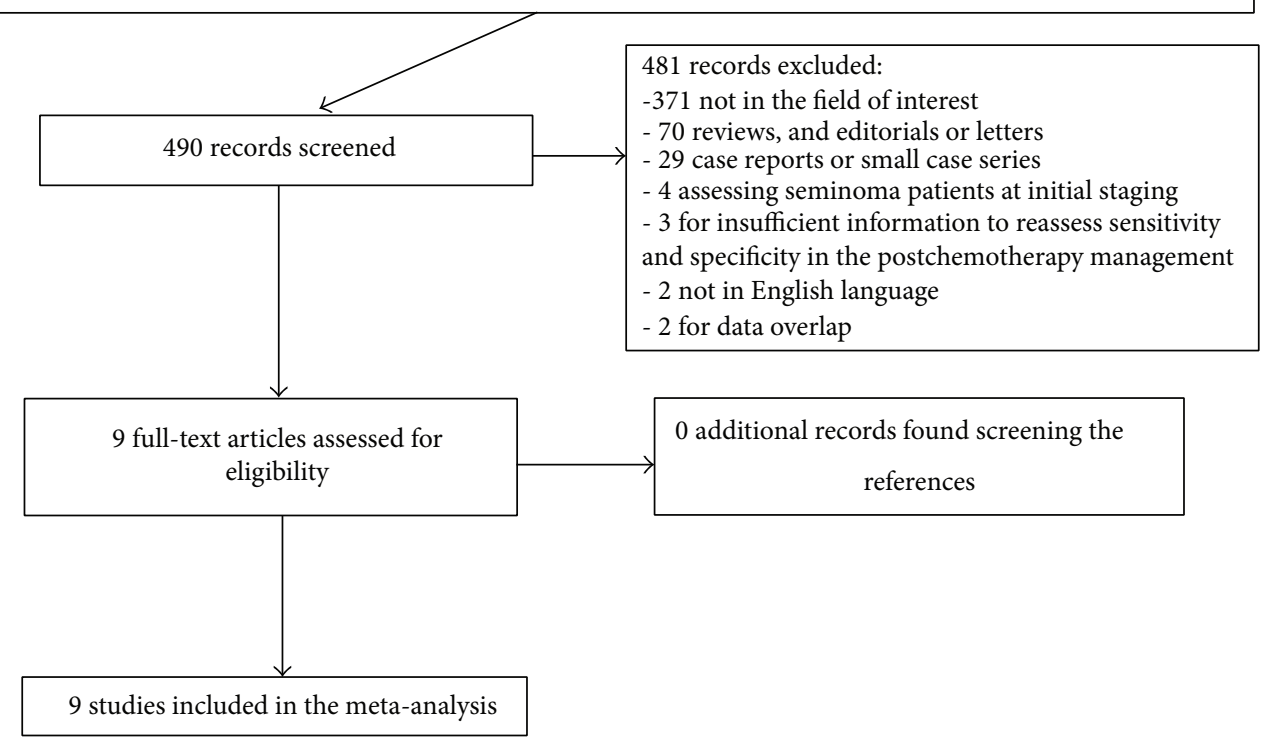

FIGURE 1: Flowchart of the search for eligible studies on the diagnostic performance of ${ }^{18} \mathrm{~F}-\mathrm{FDG}$-PET or PET/CT in the postchemotherapy management of patients with seminoma.

TABLE 1: Basic study and patient characteristics.

\begin{tabular}{|c|c|c|c|c|c|c|c|}
\hline Authors & Year & Country & Study design & $\begin{array}{l}\text { Mean age } \\
\text { (years) }\end{array}$ & $\begin{array}{c}{ }^{18} \text { F-FDG } \\
\text { PET or } \\
\text { PET/CT } \\
\text { scans } \\
\end{array}$ & $\begin{array}{l}\text { Scans with } \\
\text { residual lesion } \\
\quad<3 \mathrm{~cm}\end{array}$ & $\begin{array}{l}\text { Scans with } \\
\text { residual lesion } \\
\quad>3 \mathrm{~cm}\end{array}$ \\
\hline Ambrosini et al. [6] & 2014 & Italy & $\begin{array}{l}\text { Retrospective and } \\
\text { monocentric }\end{array}$ & 37.7 & $45^{*}$ & NR & NR \\
\hline Bachner et al. [7] & 2012 & $\begin{array}{l}\text { Several European } \\
\text { countries and Canada }\end{array}$ & $\begin{array}{l}\text { Retrospective and } \\
\text { multicentric }\end{array}$ & NR & 127 & 54 & 73 \\
\hline Siekiera et al. [8] & 2012 & Poland & $\begin{array}{l}\text { Retrospective and } \\
\text { monocentric }\end{array}$ & NR & 37 & 20 & 17 \\
\hline Hinz et al. [9] & 2008 & Germany & $\begin{array}{l}\text { Prospective and } \\
\text { multicentric }\end{array}$ & 42 & 20 & 8 & 12 \\
\hline Lewis et al. [10] & 2006 & USA & $\begin{array}{l}\text { Retrospective and } \\
\text { monocentric }\end{array}$ & NR & 24 & 13 & 11 \\
\hline De Santis et al. [11] & 2004 & Austria and Germany & $\begin{array}{l}\text { Prospective and } \\
\text { multicentric }\end{array}$ & NR & 56 & 37 & 19 \\
\hline Spermon et al. [12] & 2002 & Netherlands & $\begin{array}{c}\mathrm{NR} \text { and } \\
\text { monocentric }\end{array}$ & 30 & 10 & 8 & 2 \\
\hline Hain et al. [13] & 2000 & UK & $\begin{array}{l}\text { Retrospective and } \\
\text { monocentric }\end{array}$ & 30 & 27 & NR & NR \\
\hline Ganjoo et al. [14] & 1999 & USA & $\begin{array}{l}\text { Prospective and } \\
\text { monocentric }\end{array}$ & 38 & 29 & 8 & 18 \\
\hline
\end{tabular}

* Only patients with seminoma who underwent PET or PET/CT for restaging or evaluation of postchemotherapy residual lesions were selected; NR: not reported.

\section{Discussion}

Our systematic review and meta-analysis assessed the diagnostic performance of ${ }^{18}$ F-FDG-PET or PET/CT in the postchemotherapy management of patients with seminoma, including evaluation of residual masses after chemotherapy and restaging. Several studies have used ${ }^{18}$ F-FDG-PET or $\mathrm{PET} / \mathrm{CT}$ in this setting reporting different values of sensitivity and specificity (Figure 2). However, some of these studies have limited power, analyzing only a relatively small number of patients. In order to derive more robust estimates of the diagnostic performance of ${ }^{18}$ F-FDG-PET or PET/CT in this 


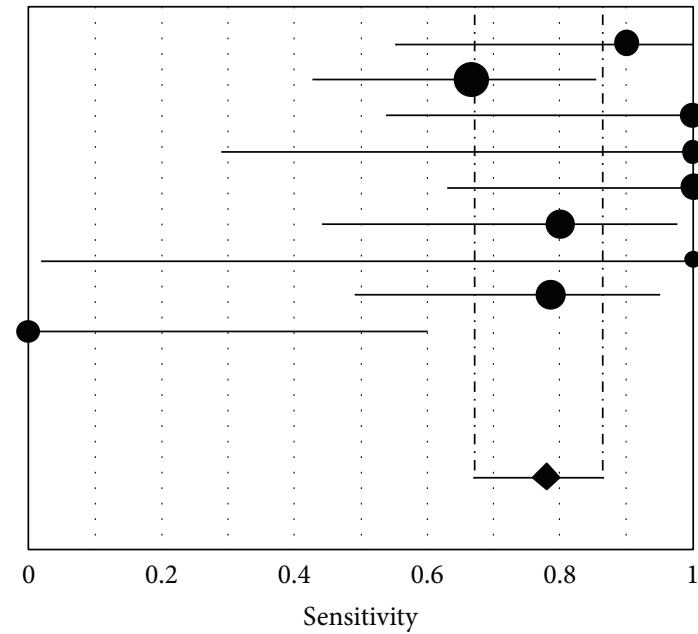

Ambrosini et al., 2014

Sensitivity (95\% CL)

Bachner et al., $2012 \quad 0.67(0.43-0.85)$

Siekiera et al., 2012

$1.00(0.54-1.00)$

Hinz et al., 2008

$1.00(0.29-1.00)$

Lewis et al., 2006

$1.00(0.63-1.00)$

de Santis et al., $2004 \quad 0.80(0.44-0.97)$

Spermon et al., $2002 \quad 1.00(0.03-1.00)$

Hain et al., 2000

$0.79(0.49-0.95)$

Ganjoo et al., 1999

$0.00(0.00-0.60)$

Pooled sensitivity $=0.78$ (0.67 to 0.87 )

$\chi^{2}=23.50 ; \mathrm{df}=8(P=0.0028)$

Inconsistency $\left(I^{2}\right)=66.0 \%$

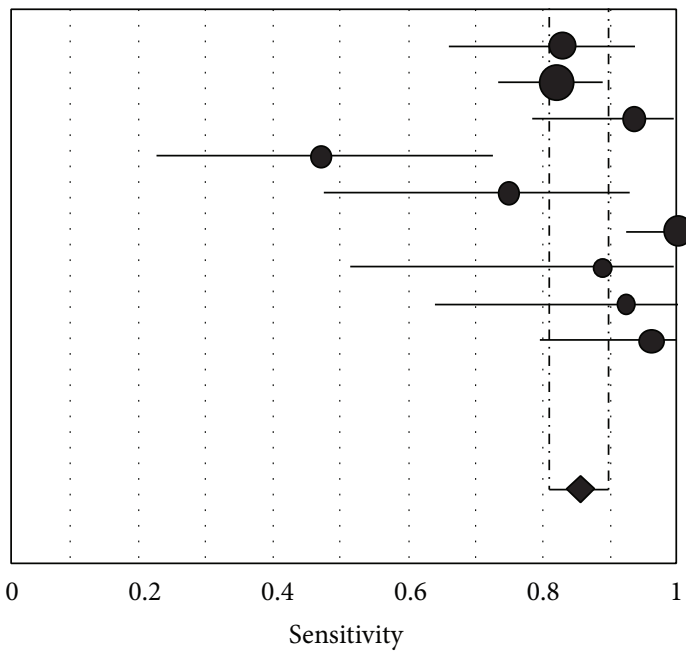

Ambrosini et al., 2014

Specificity $(95 \% \mathrm{CL})$

Bachner et al., 2012

$0.83(0.66-0.93)$

Siekiera et al., 2012

$0.82(0.73-0.89)$

Hinz et al., 2008

$0.94(0.97-0.99)$

Lewis et al., 2006

$0.47(0.23-0.72)$

de Santis et al., 2004

$0.75(0.48-0.93)$

Spermon et al., 2002

$1.00(0.92-1.00)$

$0.89(0.52-1.00)$

Hain et al., 2000

$0.92(0.64-1.00)$

Ganjoo et al., 1999

$0.96(0.80-1.00)$

Pooled sensitivity $=0.86$ (0.81 to 0.89$)$

$\chi^{2}=36.14 ; \mathrm{df}=8(P=0.0000)$

Inconsistency $\left(I^{2}\right)=77.9 \%$

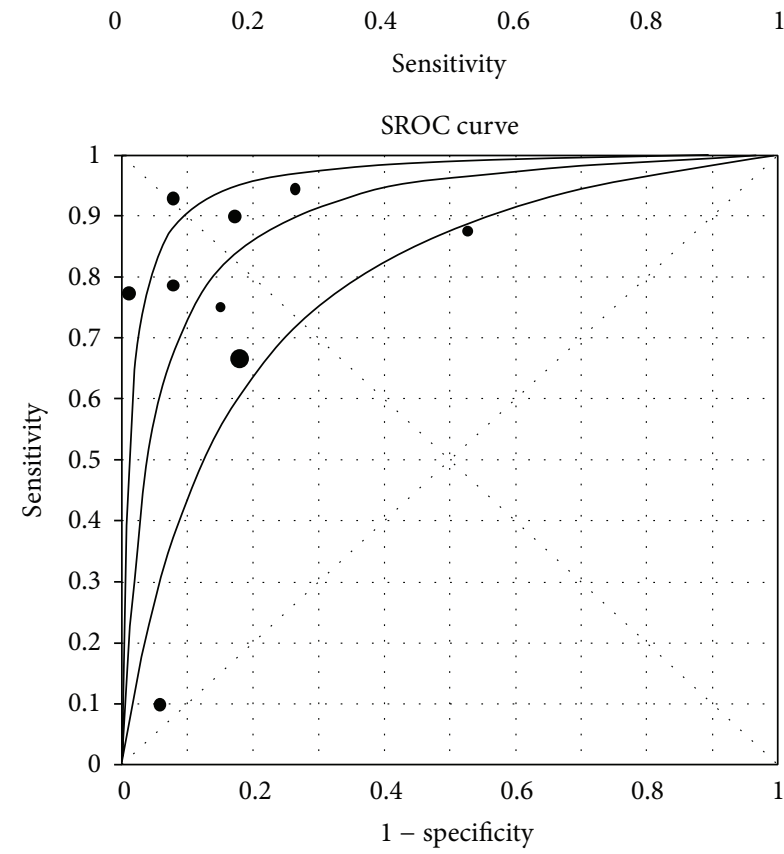

Symmetric SROC

$\mathrm{AUC}=0.9012$

SE $($ AUC $)=0.0346$

$Q^{*}=0.8326$

$\operatorname{SE}\left(Q^{*}\right)=0.0371$

FIGURE 2: Plots of pooled sensitivity and specificity and summary ROC curve of ${ }^{18} \mathrm{~F}$-FDG-PET or PET/CT in the postchemotherapy management of patients with seminoma. The area under the summary ROC curve (0.90) demonstrates that ${ }^{18}$ F-FDG-PET and PET/CT are accurate methods in this setting. 
TABLE 2: Technical aspects of ${ }^{18} \mathrm{~F}-\mathrm{FDG}$ PET and PET/CT in the included studies.

\begin{tabular}{|c|c|c|c|c|}
\hline Authors & Device & $\begin{array}{l}{ }^{18} \mathrm{~F}-\mathrm{FDG} \text { mean injected } \\
\text { dose }\end{array}$ & $\begin{array}{c}\text { Time between }{ }^{18} \mathrm{~F}-\mathrm{FDG} \\
\text { injection and image } \\
\text { acquisition }\end{array}$ & Image analysis \\
\hline Ambrosini et al. [6] & $\mathrm{PET} / \mathrm{CT}$ & $3-5.7 \mathrm{MBq} / \mathrm{kg}$ & $60 \mathrm{~min}$ & Visual \\
\hline Bachner et al. [7] & PET & NR & NR & Visual \\
\hline Siekiera et al. [8] & $\mathrm{PET} / \mathrm{CT}$ & NR & NR & Visual \\
\hline Hinz et al. [9] & PET & NR & $45-60 \mathrm{~min}$ & $\begin{array}{c}\text { Visual and } \\
\text { semiquantitative }\end{array}$ \\
\hline Lewis et al. [10] & PET & NR & NR & Visual \\
\hline De Santis et al. [11] & PET & $370 \mathrm{MBq}$ & $>45 \min$ & Visual \\
\hline Spermon et al. [12] & PET & $200-220 \mathrm{MBq}$ & $60 \mathrm{~min}$ & $\begin{array}{c}\text { Visual and } \\
\text { semiquantitative }\end{array}$ \\
\hline Hain et al. [13] & PET & $320 \mathrm{MBq}$ & NR & Visual \\
\hline Ganjoo et al. [14] & PET & $370 \mathrm{MBq}$ & $60 \mathrm{~min}$ & $\begin{array}{c}\text { Visual and } \\
\text { semiquantitative }\end{array}$ \\
\hline
\end{tabular}

NR: not reported; PET: positron emission tomography; CT: computed tomography.

TABLE 3: Diagnostic accuracy data of ${ }^{18}$ F-FDG PET and PET/CT on a per examination-based analysis.

\begin{tabular}{|c|c|c|c|c|c|c|c|c|c|c|c|c|}
\hline \multirow{3}{*}{ Author } & \multirow{2}{*}{\multicolumn{4}{|c|}{$\begin{array}{l}\text { Posttherapy evaluation, } \\
\text { surveillance, or restaging }\end{array}$}} & \multicolumn{7}{|c|}{$\begin{array}{l}\text { Subgroup analyses about } \\
\text { recurrent/residual masses at CT }\end{array}$} & \multirow[b]{3}{*}{$\mathrm{TN}$} \\
\hline & & & & & \multicolumn{4}{|c|}{ Lesions $<3 \mathrm{~cm}$} & \multicolumn{3}{|c|}{ Lesions $>3 \mathrm{~cm}$} & \\
\hline & $\mathrm{TP}$ & $\mathrm{FP}$ & FN & $\mathrm{TN}$ & $\mathrm{TP}$ & FP & FN & $\mathrm{TN}$ & $\mathrm{TP}$ & $\mathrm{FP}$ & $\mathrm{FN}$ & \\
\hline Ambrosini et al. [6] & 9 & 6 & 1 & 29 & NR & NR & NR & NR & NR & NR & NR & NR \\
\hline Bachner et al. [7] & 14 & 19 & 7 & 87 & 3 & 8 & 4 & 39 & 11 & 11 & 3 & 48 \\
\hline Siekiera et al. [8] & 6 & 2 & 0 & 29 & 0 & 1 & 0 & 19 & 6 & 1 & 0 & 8 \\
\hline Hinz et al. [9] & 3 & 9 & 0 & 8 & 2 & 3 & 0 & 3 & 1 & 6 & 0 & 5 \\
\hline Lewis et al. [10] & 8 & 4 & 0 & 12 & 1 & 2 & 0 & 10 & 7 & 2 & 0 & 2 \\
\hline De Santis et al. [11] & 8 & 0 & 2 & 46 & 1 & 0 & 2 & 34 & 7 & 0 & 0 & 12 \\
\hline Spermon et al. [12] & 1 & 1 & 0 & 8 & 0 & 0 & 0 & 8 & 1 & 1 & 0 & 0 \\
\hline Hain et al. [13] & 11 & 1 & 3 & 12 & NR & NR & NR & NR & NR & NR & NR & NR \\
\hline Ganjoo et al. [14] & 0 & 1 & 4 & 24 & 0 & 1 & 2 & 5 & 0 & 0 & 1 & 17 \\
\hline
\end{tabular}

NR: not reported; TP: true positive; FP: false positive; FN: false negative; TN: true negative.

setting we have pooled published studies [25]. A systematic review process was adopted in ascertaining studies, thereby avoiding selection bias. Furthermore, the quality of the included studies was assessed by using the 2011 Oxford Center for Evidence-Based Medicine checklist for diagnostic studies (Table 4).

A previous meta-analysis in German language evaluated the diagnostic value of ${ }^{18} \mathrm{~F}$-FDG-PET in the assessment of residual tumors after systemic treatment of metastatic seminoma including 5 studies only (130 patients) [26]. Conversely, our updated pooled analysis includes 9 articles (375 examination), thus providing more robust results on a statistical point of view.

Pooled results of our analysis indicate that ${ }^{18} \mathrm{~F}$-FDG-PET and PET/CT have good sensitivity (78\%), specificity (86\%), and accuracy (84\%) in the postchemotherapy management of patients with seminoma. ${ }^{18}$ F-FDG-PET and PET/CT have a low positive predictive value $(58 \%)$ but a very high negative predictive value (94\%) for this indication. Furthermore, the value of the AUC (0.90) demonstrates that ${ }^{18}$ F-FDG-PET and PET/CT are accurate diagnostic methods in this setting.

Our subgroup analyses considering the size of residual/recurrent lesions at CT after chemotherapy demonstrate that the sensitivity of ${ }^{18}$ F-FDG-PET and PET/CT significantly increases in patients with residual/recurrent lesions $>3 \mathrm{~cm}$ compared to those with residual/recurrent lesions $<3 \mathrm{~cm}$ (89\% versus $47 \%$, resp.), whereas the specificity slightly decreases ( $81 \%$ versus $89 \%$, resp.). The overall accuracy of ${ }^{18} \mathrm{~F}-\mathrm{FDG}-\mathrm{PET}$ and $\mathrm{PET} / \mathrm{CT}$ is superior in assessing resid$\mathrm{ual} /$ recurrent lesions $>3 \mathrm{~cm}$ compared to those $<3 \mathrm{~cm}$. On the other hand, it should be underlined that ${ }^{18}$ F-FDG-PET may early detect relapse in the postchemotherapy management of seminoma patients without abnormal findings at CT as reported in some articles $[6,13]$, because functional abnormalities evaluated by ${ }^{18}$ F-FDG-PET may precede morphological changes.

Performing subgroup analyses for different lesion size of residual/recurrent lesions has substantially great merit to 


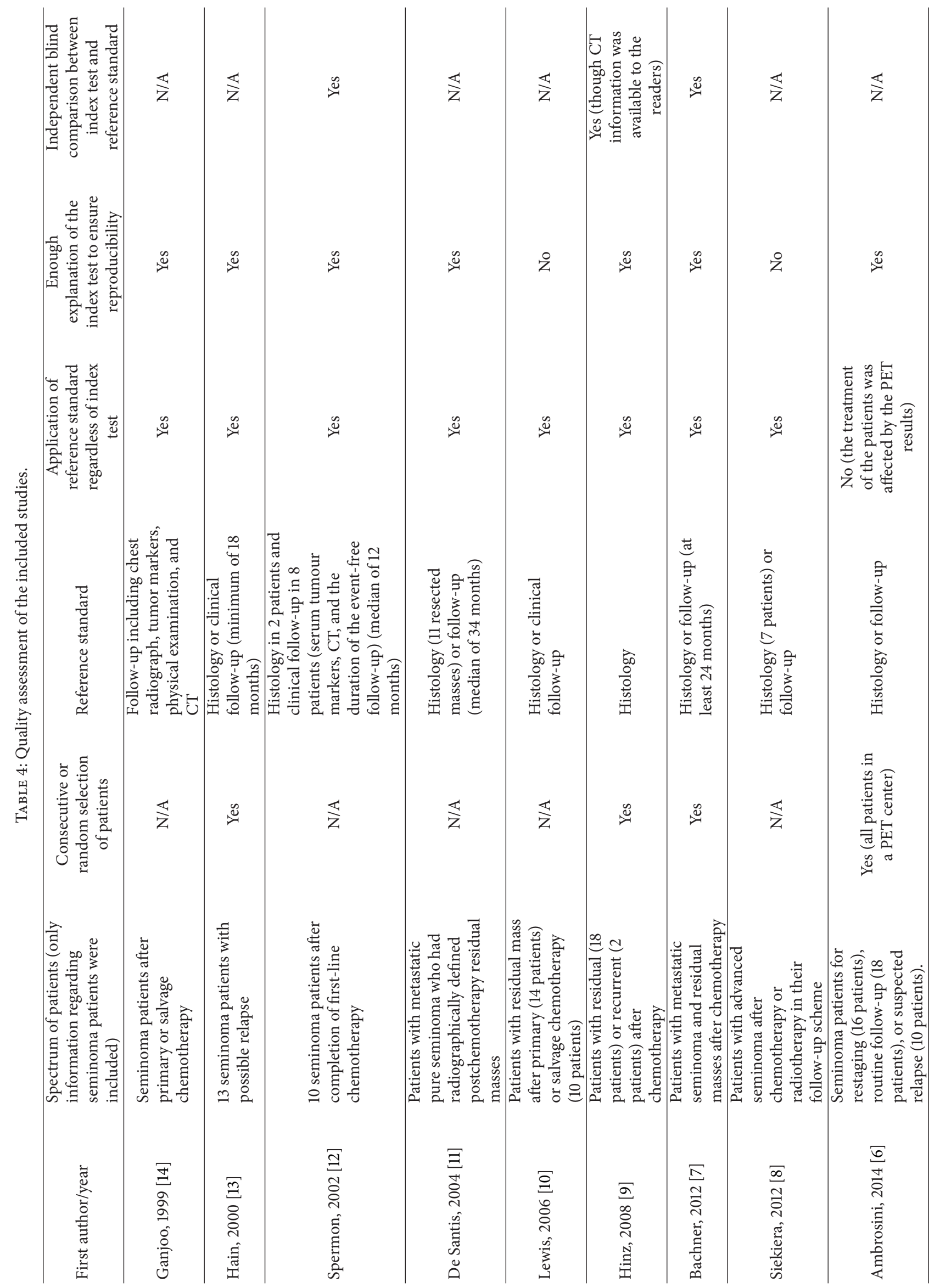




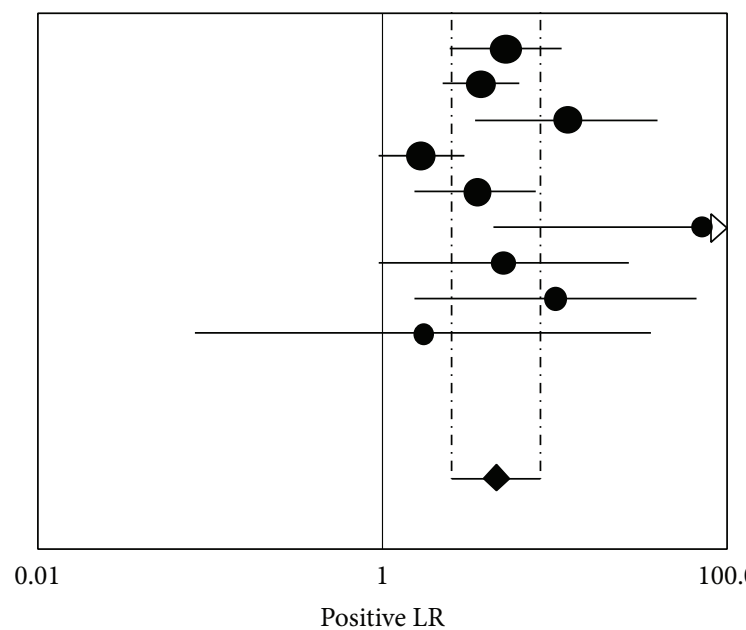

Positive LR (95\% CL)

Ambrosini et al., $2014 \quad 5.25$ (2.46-11.19)

Bachner et al., $2012 \quad 3.72(2.24-6.18)$

Siekiera et al., $2012 \quad 11.89(3.55-39.77)$

Hinz et al., $2008 \quad 1.66(0.93-2.94)$

Lewis et al., $2006 \quad 3.57(1.59-8.00)$

de Santis et al., $2004 \quad 72.64(4.53-1.165,68)$

Spermon et al., $2002 \quad 5.00(0.93-26.79)$

Hain et al., $2000 \quad 10.21(1.52-68.49)$

Ganjoo et al., $1999 \quad 1.73(0.08-36.75)$

Random effects model

Pooled positive $\mathrm{LR}=4.59$ (2.55 to 8.25 )

Cochran's $Q=22.63 ; \mathrm{df}=8(P=0.0039)$

Inconsistency $\left(I^{2}\right)=64.6 \%$

$\tau^{2}=0.4152$

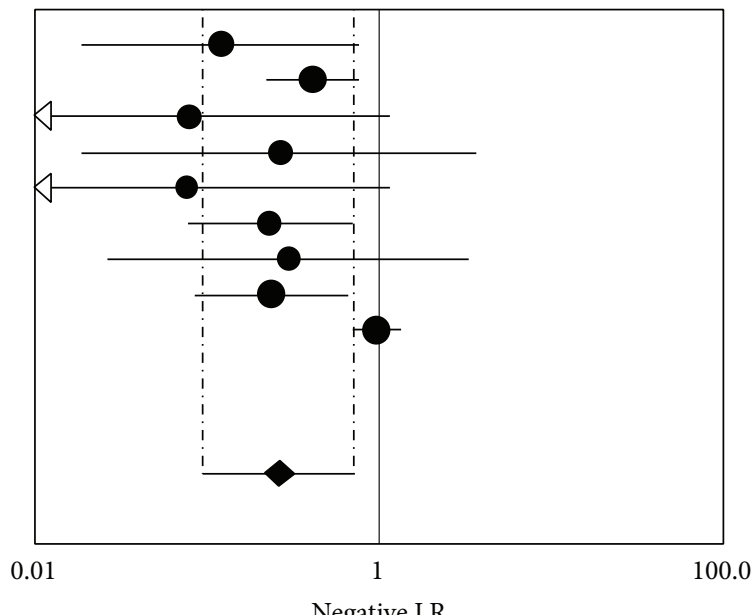

Negative LR (95\% CL)

Ambrosini et al., 2014

$0.12(0.02-0.78)$

Bachner et al., 2012

$0.41(0.22-0.75)$

Siekiera et al., 2012

$0.08(0.01-1.12)$

Hinz et al., 2008

$0.26(0.02-3.70)$

Lewis et al., 2006

$0.08(0.01-1.13)$

de Santis et al, 2004

$0.23(0.08-0.68)$

Spermon et al., 2002

$0.29(0.03-3.29)$

Hain et al., 2000

$0.23(0.08-0.64)$

Ganjoo et al., 1999

$0.96(0.70-1.30)$

Random effects model

Pooled negative $\mathrm{LR}=0.26(0.09$ to 0.71$)$

Cochran's $Q=60.62 ; \mathrm{df}=8(P=0.0000)$

Inconsistency $\left(I^{2}\right)=86.8 \%$

$\tau^{2}=1.6377$

Negative LR

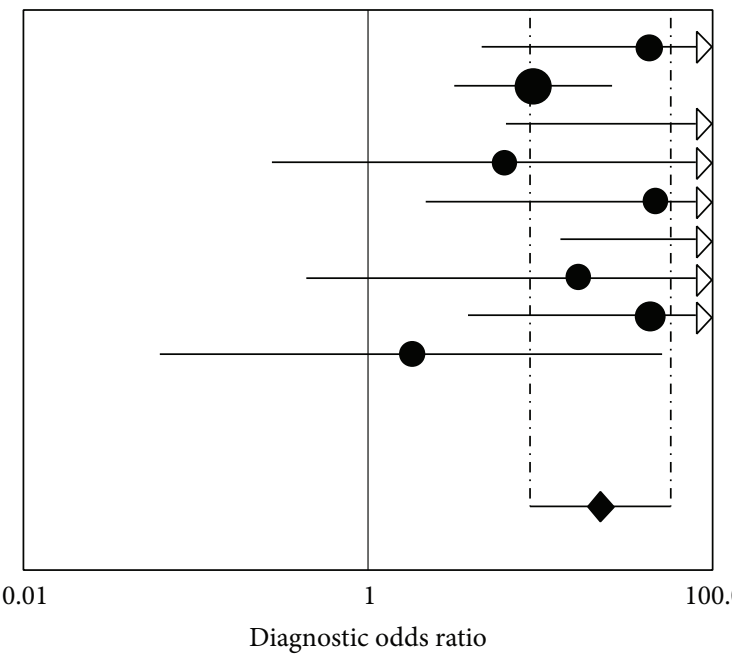

Diagnostic OR (95\% CL)

Ambrosini et al., $2014 \quad 43.50$ (4.61-410.75)

Bachner et al., $2012 \quad 9.16(3.26-25.76)$

Siekiera et al., $2012 \quad 153.40(6.55-3.590,15)$

Hinz et al., $2008 \quad 6.26(0.28-139.63)$

Lewis et al., $2006 \quad 47.22(2.24-996.03)$

de Santis et al., $2004 \quad 316.20(13.92-7.183,43)$

Spermon et al., $2002 \quad 17.00(0.45-648.21)$

Hain et al., $2000 \quad 44.00(3.97-488.19)$

Ganjoo et al., $1999 \quad 1.81(0.06-51.97)$

Random effects model

Pooled diagnostic odds ratio $=22.71(8.79$ to 58.68$)$

Cochran's $Q=10.50 ; \mathrm{df}=8(P=0.2317)$

Inconsistency $\left(I^{2}\right)=23.8 \%$

$\tau^{2}=0.4805$

FIGURE 3: Pooled positive likelihood ratio (LR), negative LR, and diagnostic odd ratio (DOR) of ${ }^{18}$ F-FDG-PET or PET/CT in the postchemotherapy management of patients with seminoma.

show as this parameter basically represents per se a source of heterogeneity among the studies. As a matter of fact, no significant heterogeneity among the studies included in the different subgroup analyses was found. Unfortunately, there were insufficient data to perform subgroup analyses taking into account the different device used (PET/CT versus PET) because only two studies assessed the diagnostic performance of ${ }^{18}$ F-FDG-PET/CT $[6,8]$. Nevertheless, a superior diagnostic performance of PET/CT compared to PET alone is expected. 


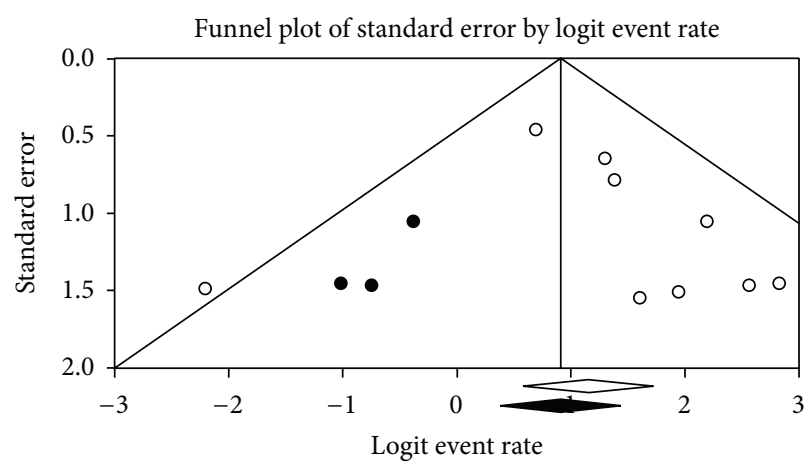

(a)

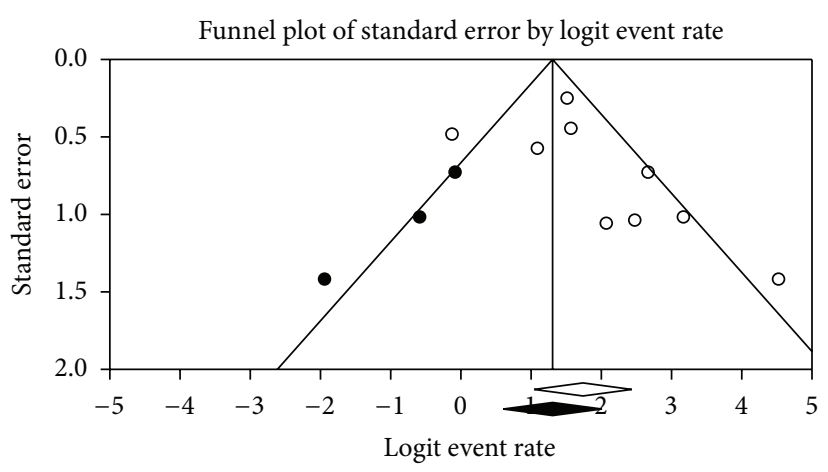

(b)

FIGURE 4: Funnel plots regarding the publication bias on the sensitivity (a) and specificity (b) of ${ }^{18}$ F-FDG-PET or PET/CT in the postchemotherapy management of patients with seminoma.

Possible sources of false-negative and false-positive results for postchemotherapy residual/recurrent seminoma at ${ }^{18}$ F-FDG-PET or PET/CT should be kept in mind. Falsenegative findings may be due to small lesions (with size below the resolution of the method) or with low proliferative activity (and consequently low ${ }^{18} \mathrm{~F}$-FDG uptake). On the other hand, the most frequent cause of false-positive findings for postchemotherapy residual/recurrent seminoma at ${ }^{18} \mathrm{~F}$ FDG-PET or PET/CT is inflammatory lesions.

The results of our analysis strengthen and support the recommendations reported in international guidelines about the usefulness of ${ }^{18} \mathrm{~F}$-FDG-PET or PET/CT in the postchemotherapy management of patients with seminoma $[27,28]$. Patients with complete response after chemotherapy do not require further treatment and are followed up. In the case of residual lesions at CT, ${ }^{18} \mathrm{~F}$-FDG-PET or PET/CT may be carried out in a minimum of 6 weeks after ending chemotherapy, in order to reduce false-positive results due to inflammation. In lesions $>3 \mathrm{~cm},{ }^{18} \mathrm{~F}$-FDG-PET or PET/CT is the recommended approach, whereas, in lesions $<3 \mathrm{~cm},{ }^{18} \mathrm{~F}-$ FDG-PET or PET/CT may be considered. Because the pooled negative predictive value is very high, ${ }^{18} \mathrm{~F}$-FDG-PET and PET/CT can replace invasive methods in the evaluation of postchemotherapy residual/recurrent lesions in patients with seminoma $[27,28]$. In this context, a negative ${ }^{18}$ F-FDG-PET warrants follow-up only avoiding inappropriate subsequent treatment (surgery, chemotherapy, or radiotherapy). In the case of a positive ${ }^{18} \mathrm{~F}$-FDG-PET, the possibility of residual seminoma is high, though a false-positive result cannot be excluded as demonstrated by its low positive predictive value.

Possible limitations of our meta-analysis could be the heterogeneity between the included studies, the publication bias, and the low number of the selected studies [25].

Heterogeneity between studies may represent a potential source of bias in a meta-analysis. This heterogeneity is likely to arise through diversity in methodological aspects between different studies (Table 2). The baseline differences among the patients in the included studies, the reference standard used, and the study quality (Table 4 ) may contribute to the heterogeneity of the results too. In our pooled analysis the included studies were statistically heterogeneous in their estimate of sensitivity and specificity. However, heterogeneity among the studies was not found performing subgroup analyses for different size of residual/recurrent lesions. In order to limit the heterogeneity we decided to include in our pooled analysis only studies which evaluated the postchemotherapy management of patients with seminoma, excluding those which described the role of ${ }^{18} \mathrm{~F}$-FDG-PET or PET/CT at initial staging.

Publication bias is a major concern in all meta-analyses as studies reporting significant findings are more likely to be published than those reporting nonsignificant results. Indeed, it is not unusual for small-sized early studies to report a positive relationship that subsequent larger studies fail to replicate. We assessed publication bias in our metaanalysis using qualitative and quantitative methods (Egger's regression and Duval and Tweedie's method). Funnel plots showed an asymmetry for both sensitivity and specificity pooling, and Duval and Tweedie's method also showed the importance of possible publication bias as estimated unbiased pooled sensitivity and specificity decreased considerably compared to the original estimates.

Only nine studies are included in the quantitative analysis and this could limit the statistical power of our meta-analysis. Some of them presented a low sample size (Table 1). Overall the quality of the included studies was moderate (Table 4). Four studies reported the consecutive recruitment of the patients and only three studies reported blind interpretation of the ${ }^{18}$ F-FDG-PET or PET/CT findings, which can introduce interpretation bias (when observers of PET or PET/CT studies had prior knowledge influencing their interpretation of the results). On the other hand, both prospective and multicentric clinical trials are available to this regard.

\section{Conclusions}

${ }^{18}$ F-FDG-PET and PET/CT were demonstrated to be accurate diagnostic imaging methods in the postchemotherapy management of patients with seminoma (in particular in patients with recurrent/residual lesions $>3 \mathrm{~cm}$ ); nevertheless possible 
sources of false-negative and false-positive results should be kept in mind. The literature focusing on the use of ${ }^{18} \mathrm{~F}$-FDG$\mathrm{PET}$ and PET/CT in this setting still remains limited and costeffectiveness analyses are warranted.

\section{Conflict of Interests}

The authors declare that there is no conflict of interests regarding the publication of this paper.

\section{Acknowledgment}

The authors have no funding.

\section{References}

[1] A. Nallu, H. D. Mannuel, and A. Hussain, "Testicular germ cell tumors: biology and clinical update," Current Opinion in Oncology, vol. 25, pp. 266-272, 2013.

[2] S. Daneshmand, P. Albers, S. D. Fosså et al., "Contemporary management of postchemotherapy testis cancer," European Urology, vol. 62, no. 5, pp. 867-876, 2012.

[3] M. L. Quek, V. Simma-Chiang, J. P. Stein, J. Pinski, D. I. Quinn, and D. G. Skinner, "Postchemotherapy residual masses in advanced seminoma: current management and outcomes," Expert Review of Anticancer Therapy, vol. 5, no. 5, pp. 869-874, 2005.

[4] G. Treglia, E. Cason, and G. Fagioli, "Recent applications of nuclear medicine in diagnostics (I part)," Italian Journal of Medicine, vol. 4, no. 2, pp. 84-91, 2010.

[5] A. Liberati, D. G. Altman, J. Tetzlaff et al., "The PRISMA statement for reporting systematic reviews and meta-analyses of studies that evaluate health care interventions: explanation and elaboration," Journal of Clinical Epidemiology, vol. 62, no. 10, pp. e1-e34, 2009.

[6] V. Ambrosini, G. Zucchini, S. Nicolini et al., " ${ }^{18}$ F-FDG PET/CT impact on testicular tumours clinical management," European Journal of Nuclear Medicine and Molecular Imaging, vol. 41, no. 4, pp. 668-673, 2014.

[7] M. Bachner, Y. Loriot, M. Gross-Goupil et al., "2- ${ }^{18}$ fluorodeoxy-D-glucose positron emission tomography (FDG-PET) for postchemotherapy seminoma residual lesions: a retrospective validation of the SEMPET trial," Annals of Oncology, vol. 23, no. 1, pp. 59-64, 2012.

[8] J. Siekiera, B. Małkowski, W. Jóźwicki et al., "Can we rely on PET in the follow-up of advanced seminoma patients?" Urologia Internationalis, vol. 88, no. 4, pp. 405-409, 2012.

[9] S. Hinz, M. Schrader, C. Kempkensteffen et al., "The role of positron emission tomography in the evaluation of residual masses after chemotherapy for advanced stage seminoma," The Journal of Urology, vol. 179, no. 3, pp. 936-940, 2008.

[10] D. A. Lewis, M. Tann, K. Kesler, A. McCool, R. S. Foster, and L. H. Einhorn, "Positron emission tomography scans in postchemotherapy seminoma patients with residual masses: a retrospective review from Indiana University Hospital," Journal of Clinical Oncology, vol. 24, no. 34, pp. e54-e55, 2006.

[11] M. de Santis, A. Becherer, C. Bokemeyer et al., "2- ${ }^{18}$ fluorodeoxy-D-glucose positron emission tomography is a reliable predictor for viable tumor in postchemotherapy seminoma: an update of the prospective multicentric SEMPET trial," Journal of Clinical Oncology, vol. 22, no. 6, pp. 1034-1039, 2004.
[12] J. R. Spermon, L. F. de Geus-Oei, L. A. L. M. Kiemeney, J. A. Witjes, and W. J. G. Oyen, "The role of ${ }^{18}$ fluoro-2-deoxyglucose positron emission tomography in initial staging and re-staging after chemotherapy for testicular germ cell tumours," BJU International, vol. 89, no. 6, pp. 549-556, 2002.

[13] S. F. Hain, M. J. O'Doherty, A. R. Timothy, M. D. Leslie, P. G. Harper, and R. A. Huddart, "Fluorodeoxyglucose positron emission tomography in the evaluation of germ cell tumours at relapse," British Journal of Cancer, vol. 83, no. 7, pp. 863-869, 2000.

[14] K. N. Ganjoo, R. J. Chan, M. Sharma, and L. H. Einhorn, "Positron emission tomography scans in the evaluation of postchemotherapy residual masses in patients with seminoma," Journal of Clinical Oncology, vol. 17, no. 11, pp. 3457-3460, 1999.

[15] Oxford Center for Evidence-Based Medicine checklist for diagnostic studies appraisal, January 2014, http://www.cebm.net/ index.aspx?o=1157.

[16] M. Egger, G. Davey Smith, M. Schneider, and C. Minder, "Bias in meta-analysis detected by a simple, graphical test," British Medical Journal, vol. 315, pp. 629-634, 1997.

[17] S. Duval and R. Tweedie, "Trim and fill: a simple funnel-plotbased method of testing and adjusting for publication bias in meta-analysis," Biometrics, vol. 56, no. 2, pp. 455-463, 2000.

[18] U. Cremerius, P. J. Effert, G. Adam et al., "FDG PET for detection and therapy control of metastatic germ cell tumor," Journal of Nuclear Medicine, vol. 39, no. 5, pp. 815-822, 1998.

[19] Y. Sugawara, K. R. Zasadny, H. B. Grossman, I. R. Francis, M. F. Clarke, and R. L. Wahl, "Germ cell tumor: differentiation of viable tumor, mature teratoma, and necrotic tissue with FDG PET and kinetic modeling," Radiology, vol. 211, no. 1, pp. 249256, 1999.

[20] P. Tsatalpas, B. Beuthien-Baumann, J. Kropp et al., "Diagnostic value of ${ }^{18} \mathrm{~F}$-FDG positron emission tomography for detection and treatment control of malignant germ cell tumors," Urologia Internationalis, vol. 68, no. 3, pp. 157-163, 2002.

[21] V. Müller-Mattheis, M. Reinhardt, C. D. Gerharz et al., "Positron-emission tomography with ${ }^{18} \mathrm{~F}$-2-fluoro-2-deoxyD-glucose $\left({ }^{18} \mathrm{FDG}-\mathrm{PET}\right)$ in the diagnosis of retroperitoneal lymph-node metastases from testicular tumors," Der Urologe A, vol. 37, no. 6, pp. 609-620, 1998.

[22] A. C. Pfannenberg, K. Oechsle, C. Kollmannsberger et al., "Early prediction of treatment response to high-dose chemotherapy in patients with relapsed germ cell tumors using $\left[{ }^{18} \mathrm{~F}\right] \mathrm{FDG}-\mathrm{PET}$, CT or MRI, and tumor marker," RöFo, vol. 176, no. 1, pp. 76-84, 2004.

[23] M. de Santis, C. Bokemeyer, A. Becherer et al., "Predictive impact of $2-{ }^{18}$ fluoro-2-deoxy-D-glucose positron emission tomography for residual postchemotherapy masses in patients with bulky seminoma," Journal of Clinical Oncology, vol. 19, no. 17, pp. 3740-3744, 2001.

[24] A. Becherer, M. de Santis, G. Karanikas et al., "FDG PET is superior to CT in the prediction of viable tumour in post-chemotherapy seminoma residuals," European Journal of Radiology, vol. 54, no. 2, pp. 284-288, 2005.

[25] G. Treglia and R. Sadeghi, "Meta-analyses and systematic reviews on PET and PET/CT in oncology: the state of the art," Clinical and Translational Imaging, vol. 1, pp. 73-75, 2013.

[26] J. Müller, A. J. Schrader, F. Jentzmik, and M. Schrader, "Assessment of residual tumours after systemic treatment of metastatic seminoma : ${ }^{18} \mathrm{~F}$-2-fluoro-2-deoxy-D-glucose positron emission tomography-meta-analysis of diagnostic value," Der Urologe $A$, vol. 50, no. 3, pp. 322-327, 2011. 
[27] J. Oldenburg, S. D. Fosså, J. Nuver et al., "Testicular seminoma and non-seminoma: ESMO Clinical Practice Guidelines for diagnosis, treatment and follow-up," Annals of Oncology, vol. 24, supplement 6, pp. vil25-vil32, 2013.

[28] NCCN guidelines for testicular cancer, January 2014, http:// www.nccn.org/professionals/physician_gls/pdf/testicular.pdf. 


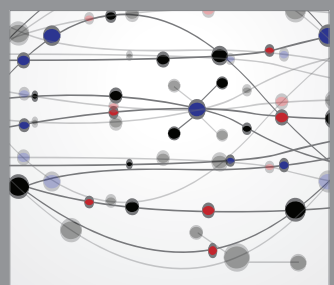

The Scientific World Journal
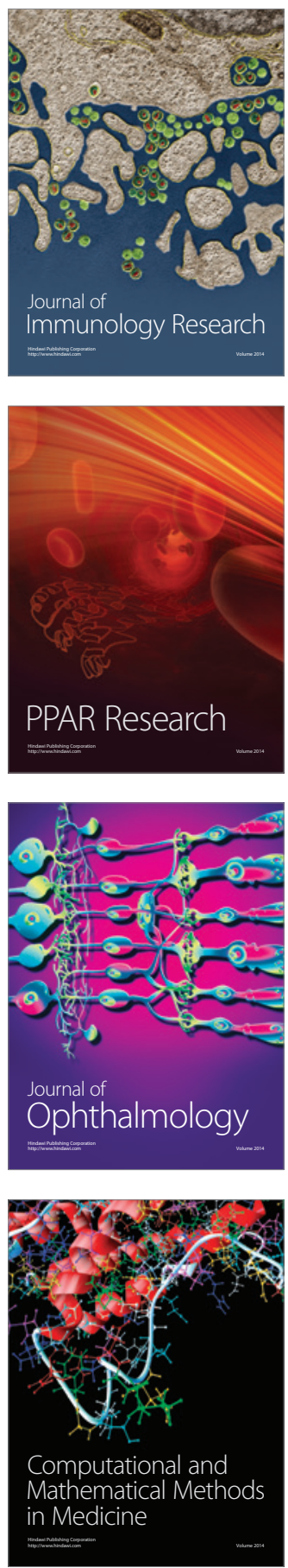

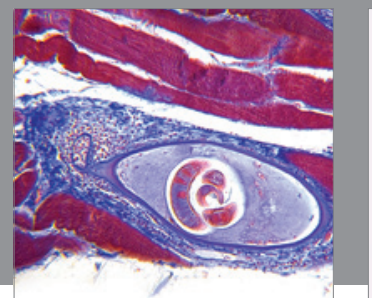

Gastroenterology

Research and Practice
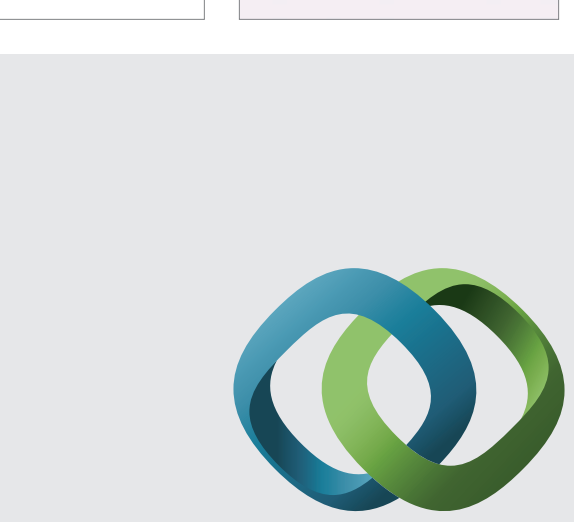

\section{Hindawi}

Submit your manuscripts at

http://www.hindawi.com
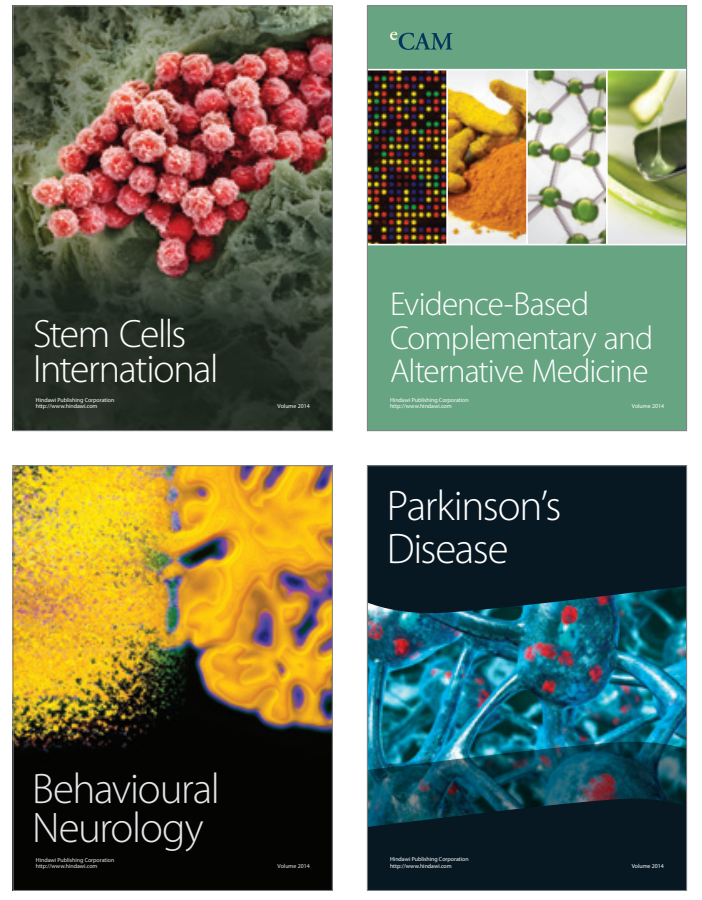
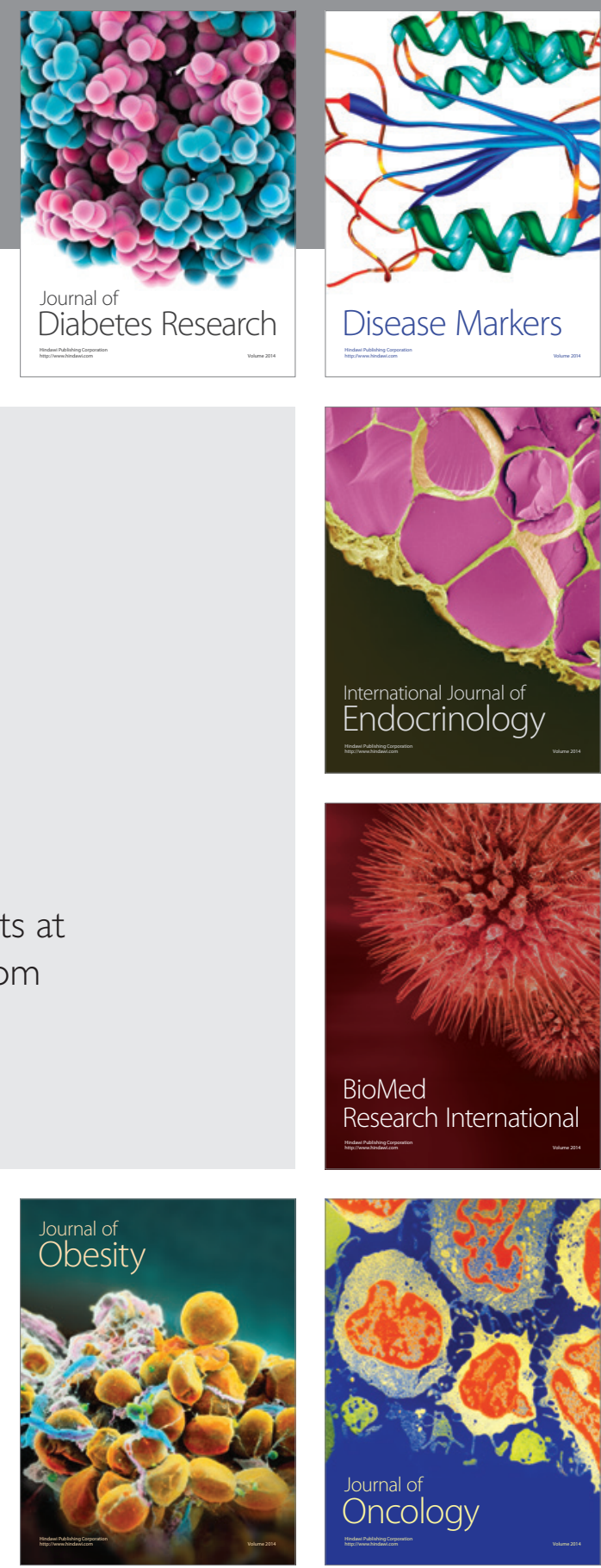

Disease Markers
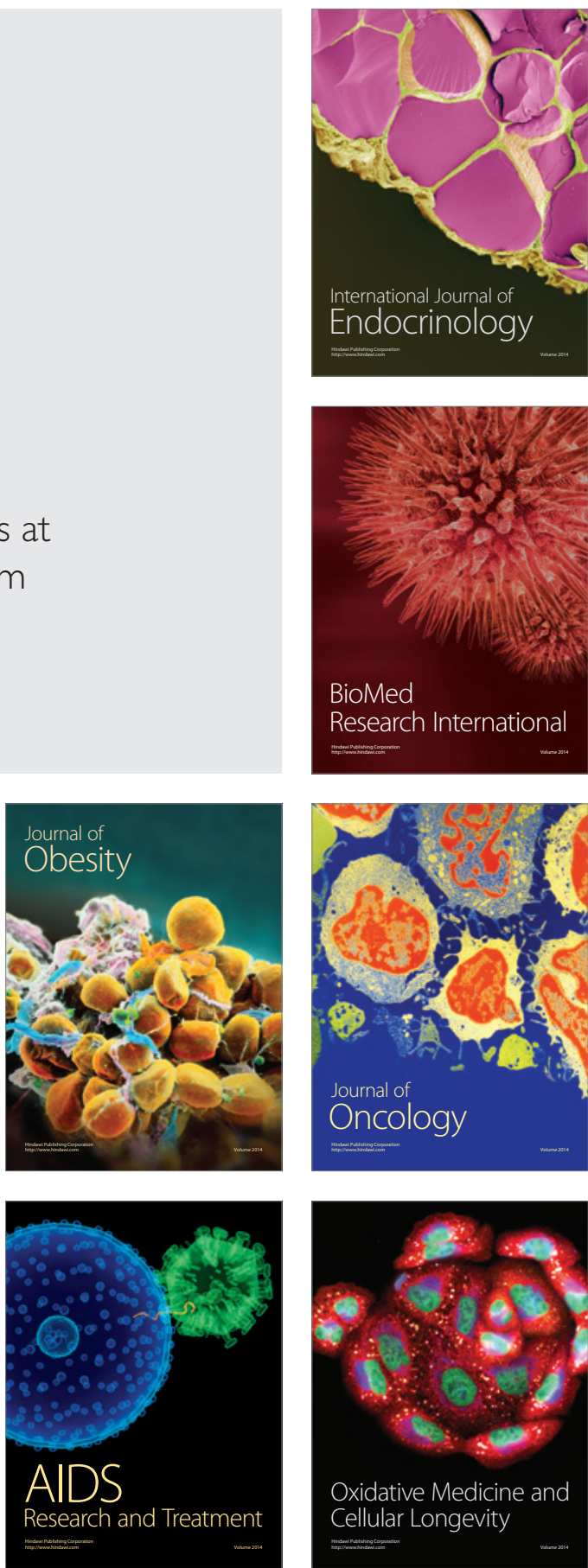